\title{
Sign Language Translation in a Healthcare Setting ${ }^{\star}$
}

\author{
Floris Roelofsen ${ }^{1}$, Lyke Esselink ${ }^{1}$, Shani Mende-Gillings ${ }^{1}$, and Anika Smeijers ${ }^{2}$ \\ 1 Institute for Logic, Language and Computation, Amsterdam, the Netherlands \\ \{f.roelofsen, l.d.esselink, s.e.mendegillings\}@uva.nl \\ 2 Amsterdam University Medical Centre, Amsterdam, the Netherlands \\ a.s.smeijers@amsterdamumc.nl
}

\begin{abstract}
Communication between healthcare professionals and deaf patients is challenging, and the current COVID-19 pandemic makes this issue even more acute. Sign language interpreters can often not enter hospitals and face masks make lipreading impossible. To address this urgent problem, we developed a system which allows healthcare professionals to translate sentences that are frequently used in the diagnosis and treatment of COVID-19 into Sign Language of the Netherlands (NGT). Translations are displayed by means of videos and avatar animations. The architecture of the system is such that it could be extended to other applications and other sign languages in a relatively straightforward way.
\end{abstract}

Keywords: text-to-sign translation · healthcare translation technology

\section{Introduction}

Communication between healthcare professionals and deaf patients is challenging [11, especially during the current COVID-19 pandemic [20. Sign language interpreters can often not enter hospitals and clinics, interpreting via video relay is not always viable, and face masks make lipreading impossible [14].

We conducted an online questionnaire to understand how these difficulties are perceived by deaf people in the Netherlands. Questions were video recorded in Sign Language of the Netherlands (NGT) and also presented in written Dutch. 179 people participated, aged 20 to 84 . The results - which will be presented in detail elsewhere - show that the general inability of healthcare professionals to communicate in sign language is indeed perceived as a very significant threat. Specifically, $88 \%$ of participants stated that they were worried about communication barriers should they need to be hospitalised with COVID-19, while, for

\footnotetext{
* We are grateful to Onno Crasborn, Manny Rayner, Marijke Scheffener, Irène Strasly, Roos Wattel, and Inge Zwitserlood for helpful discussions at various stages of this project. Special thanks to Bastien David, John Glauert, and Richard Kennaway for their very generous help with JASigning and SiGML. Finally, we gratefully acknowledge financial support from the Netherlands Organisation for Innovation in Healthcare (ZonMw, grant number 10430042010027) and the European Research Council (ERC, grant number 680220).
} 
comparison, only $33 \%$ stated that they were worried about the fact that friends and relatives would not be allowed to visit them in the hospital.

To address this problem, we have developed a modular system which allows healthcare professionals to translate phrases that are frequently used in the diagnosis and treatment of hospital patients, in particular phrases related to COVID-19, from Dutch or English into NGT. For a limited number of sentences, translations are displayed by means of pre-recorded videos. In addition, the system is able to generate translations that are displayed by means of a signing avatar. The present paper focuses on describing the latter part of the system.

Although we have concentrated on NGT as the target sign language, the problem we aim to address manifests itself worldwide ${ }^{1}$ Therefore, in contrast to most existing text-to-sign translation systems (which are tailor-made for a specific target sign language and not easily portable to other languages, see Sections 3 and 4 , we have aimed to design the system in such a way that it could be extended to include other source and target languages in a relatively straightforward way.

We should emphasise that a qualified human sign language interpreter should, whenever available, always be preferred over a machine translation system. Still, it is worth investigating the extent to which a machine translation system can be of help in situations in which a human interpreter cannot be employed, especially in the medical setting where effective, instantaneous communication between healthcare professionals and patients can be of critical importance.

\section{$2 \quad$ Brief background on sign languages}

Evidently, we cannot provide a comprehensive overview here of the linguistic properties of sign languages in general (see, e.g., [1]), nor of NGT in particular (see [19]). We will, however, highlight some important features which any textto-sign translation system needs to take into account.

First of all, sign languages have naturally evolved in deaf communities around the world. This means that, contrary to a rather common misconception, there is not a single, universal sign language used by all deaf people worldwide, but many different sign languages, just as there are many different spoken languages.

Second, deaf people often have great difficulties processing spoken language even if presented in written form. The median reading level of deaf adolescents when finishing high-school is comparable to that of 8-year-old hearing children 2717. This may be surprising at first sight but not so much upon reflection. Imagine what it would be like as a native speaker of, say, English, to learn Hebrew or Thai just by looking at the characters, without being told how these characters are pronounced. Thus, for healthcare professionals to communicate with deaf patients exclusively through written text would not be satisfactory. This is especially true in a medical setting, where it is critical to avoid miscom-

${ }^{1}$ The World Federation of the Deaf estimates that there are around 70 million deaf people around the world (see https://wfdeaf.org/). 
munication, to obtain reliable informed consent for interventions, and to foster an environment in which patients feel maximally safe.

Third, there is generally no direct correspondence between the sign language used in a given country and the spoken language used in that same country. For instance, while English is the mainstream spoken language both in the US and in the UK, American Sign Language (ASL) and British Sign Language (BSL) differ considerably from each other, as well as from spoken English. Such differences do not only pertain to the lexicon, but also to grammatical features such as word order. This means in particular that, to translate a sentence from English to ASL or BSL it does not suffice to translate every word in the sentence into the corresponding sign in ASL/BSL and then put these signs together in the same order as the words in the English sentence.

Fourth, signs are generally not just articulated with the hands, but often also involve facial expressions and/or movements of the head, mouth, shoulders, or upper body. These are referred to as the non-manual components of a sign. A text-to-sign translation system has to take both manual and non-manual components of signs into account.

Fifth, related to the previous point, non-manual elements are not only part of the lexical make-up of many signs, but are also often used to convey certain grammatical information (comparable to intonation in spoken languages). For instance, raised eyebrows may indicate that a given sentence is a question rather than a statement, and a head shake expresses negation. Such non-manual grammatical markers are typically 'supra-segmental', meaning that they do not co-occur with a single lexical sign but rather span across a sequence of signs in a sentence. Sign language linguists use so-called glosses to represent sign language utterances. For instance, the gloss in (1) represents the NGT translation of the question Are you going on holiday?

$\frac{\text { brow raise }}{\text { YOU HOLIDAY GO }}$

Lexical signs are written in small-caps. They always involve a manual component and often non-manual components as well. The upper tier shows non-manual grammatical markers, and the horizontal line indicates the duration of these nonmanual markers. In this case, 'brow raise' is used to indicate that the utterance is a question. A text-to-sign translation system should be able to integrate nonmanual elements that convey grammatical information with manual and nonmanual elements that belong to the lexical specification of the signs in a given sentence 28 . This means that a system which translates sentences word by word, even if it re-orders the corresponding signs in accordance with the word order rules of the target sign language, cannot be fully satisfactory. More flexibility is needed: word by word translation can be a first step, but the corresponding signs as specified in the lexicon, must generally be adapted when forming part of a sentence to incorporate non-manual markers of grammatical information. 


\section{Sign synthesis}

A crucial prerequisite for text-to-sign translation is sign synthesis: the ability to create sign language avatar animations. Broadly speaking there are two ways to achieve this: key-frame animation (e.g., 7]) and motion capture (e.g., 12]).

While motion capture makes it possible to obtain a library of high-quality animations for lexical signs, a disadvantage of this technique is that animations for lexical signs obtained in this way are difficult to modify so as to incorporate non-manual grammatical markers [5]. In principle, the same problem also applies to libraries of lexical signs obtained by means of key-frame animation. However, in this case, there is a promising strategy to overcome the problem. Namely, rather than directly animating each lexical sign, it is possible to generate keyframe animations of lexical signs procedurally from structured specifications of the phonetic properties of these signs 9 . Such phonetic properties include (but are not limited to) the initial location, shape and orientation of the hands, possibly movements of the hands and other body parts, and facial expressions. Several formalisms have been developed to specify the phonetic properties of signs in a structured, computer-readable fashion (see [5] for an overview). Arguably the most extensively developed and most widely used formalism is the Sign Gesture Markup Language (SiGML) 913], which is based on the HamNoSys notation originally developed for the annotation of sign language corpora 22 15. For illustration, our SiGML encoding of the NGT sign wHAT is given in Figure1. As can be seen in the figure, both manual components (handshape, location, movement) and non-manual features (mouth, face, head) are encoded.

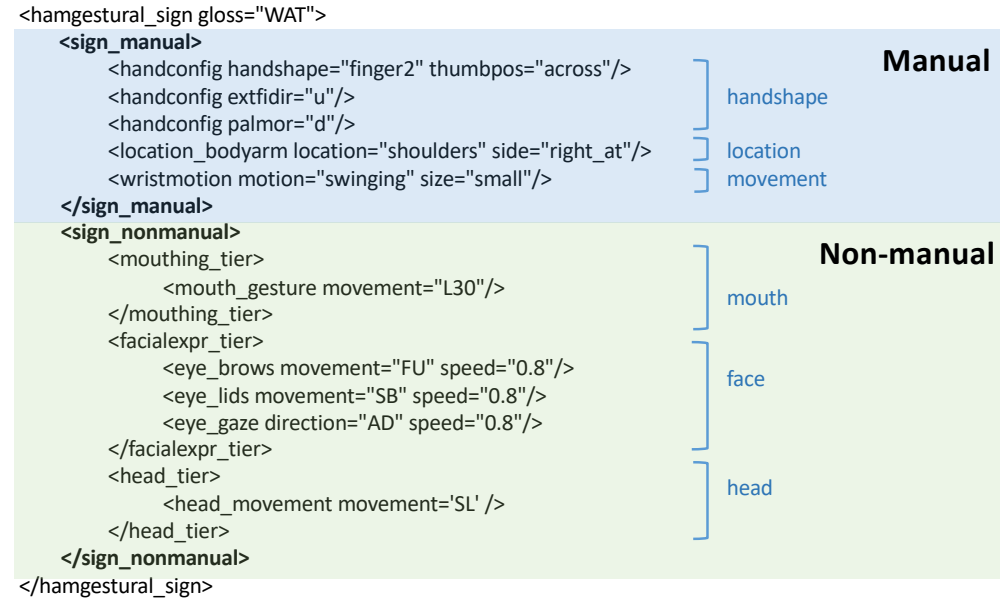

Fig. 1. SiGML encoding of the NGT sign WAT ('what').

SiGML specifications can be converted into key-frame animations by the JASigning avatar engine 9|18|16. This approach makes it possible, in principle, 
to integrate non-manual grammatical markers with the lexical signs that make up a sentence, although such functionality has not yet been thoroughly implemented in systems based on SiGML and JASigning to our knowledge.

Given these considerations, we opted to use SiGML and JASigning as a basis for sign language synthesis, and to implement a new functionality to automate the integration of non-manual grammatical markers with lexical signs. A basic library of SiGML specifications of around 2000 lexical signs in NGT was already compiled in the course of previous projects $([10$, see also [182310]). While we have had to extend this library with healthcare-related as well as some generalpurpose signs, the availability of an initial repertoire of signs encoded in SiGML was essential for a timely development of the system.

\section{Text-to-sign translation: A modular approach}

In text-to-sign translation, two general approaches can be distinguished, differing mainly in the type of intermediate representation that is employed in going from text to sign.

In the first approach, which we will refer to as the gloss approach, a given input sentence is transformed into a gloss of the corresponding sign language utterance. Next, based on this gloss representation, an avatar animation is generated.

$$
\text { Gloss approach: } \quad \text { text } \Longrightarrow \text { gloss } \Longrightarrow \text { animation }
$$

This approach is taken, for instance, by HandTalk, a Brazilian company that provides an automated text-to-sign translation service with Brazilian Portuguese and English as possible source languages, and ASL as well as Brazilian Sign Language as possible target languages. HandTalk uses machine learning techniques to map input texts to the corresponding glosses, and a combination of key-frame animation and motion capture techniques to generate animations based on a given gloss.

In the second approach, which we refer to as the phonetic approach, the given input sentence is transformed into a sequence of phonetic representations of signs. Next, based on these phonetic representations, an avatar animation is generated.

\section{(3) Phonetic approach: $\quad$ text $\Longrightarrow$ phonetic rep. $\Longrightarrow$ animation}

This approach has been taken in work based on SiGML and JASigning (see, e.g., 3018 23 2 386 ]). Unlike in the gloss approach, applying machine learning techniques to carry out the first step, from text to phonetic representations, is not feasible because it would require the availability of large parallel corpora of texts and the corresponding phonetic sign representations, which do not exist and would be very costly to create. The process of manually generating phonetic representations is highly time-consuming and requires expert knowledge of SiGML or a similar formalism. Rayner et al. 24] have created a framework 
to ease this process, which is especially helpful if the sentences that need to be translated are all variations of a limited set of templates. For instance, the framework has been used successfully to develop an application for translating railway announcements $[6]$. In less restricted domains, however, generating phonetic representations still requires expert knowledge of SiGML or similar formalisms and remains very time-intensive.

The gloss approach and the phonetic approach have complementary pros and cons. An advantage of the gloss approach is that it enables the use of machine learning technology to carry out the first part of the translation process. Disadvantages are that (i) the animation of each individual sign involves a lot of manual work, (ii) grammatical non-manual elements cannot be straightforwardly integrated with lexical signs, and (iii) all components of the system are tailor-made for a particular target sign language, i.e., no part of the system can be re-used when a new target language is considered. In particular, since no gloss-based system currently exists for NGT, this approach was not viable for our purposes.

Advantages of the phonetic approach are that (i) grammatical non-manual features can in principle be integrated with lexical signs (though this possibility remains largely unexplored) and (ii) part of the system, namely the software that generates avatar animations based on phonetic representations (i.e., JASigning or a similar avatar engine) is not language-specific and can be used for any target sign language. The main disadvantage is that the initial step from text to phonetic representations involves a lot of manual work.

Given these considerations, we propose a modular approach, which employs both a gloss representation and a phonetic representation in going from a given input text to an avatar animation of the corresponding sign language utterance. As depicted in Figure 2, our modular approach breaks the translation process up into three steps:

\section{Gloss translation}

In this step, the given Dutch or English input sentence is mapped to a gloss representation of the corresponding NGT sentence.

\section{Phonetic encoding}

In this step, the NGT gloss is transformed into a computer-readable phonetic representation, in our case formulated in SiGML.

\section{Animation}

In this step, an avatar animation is generated based on the given phonetic representation.

Consider, for instance, the Dutch/English input sentence in (4):

(4) Waar doet het pijn?

Where does it hurt?

The first step is to convert this sentence into the corresponding NGT gloss in (5), where 'whq' stands for the non-manual marking that is characteristic for constituent questions in NGT. While empirical studies have found quite some 


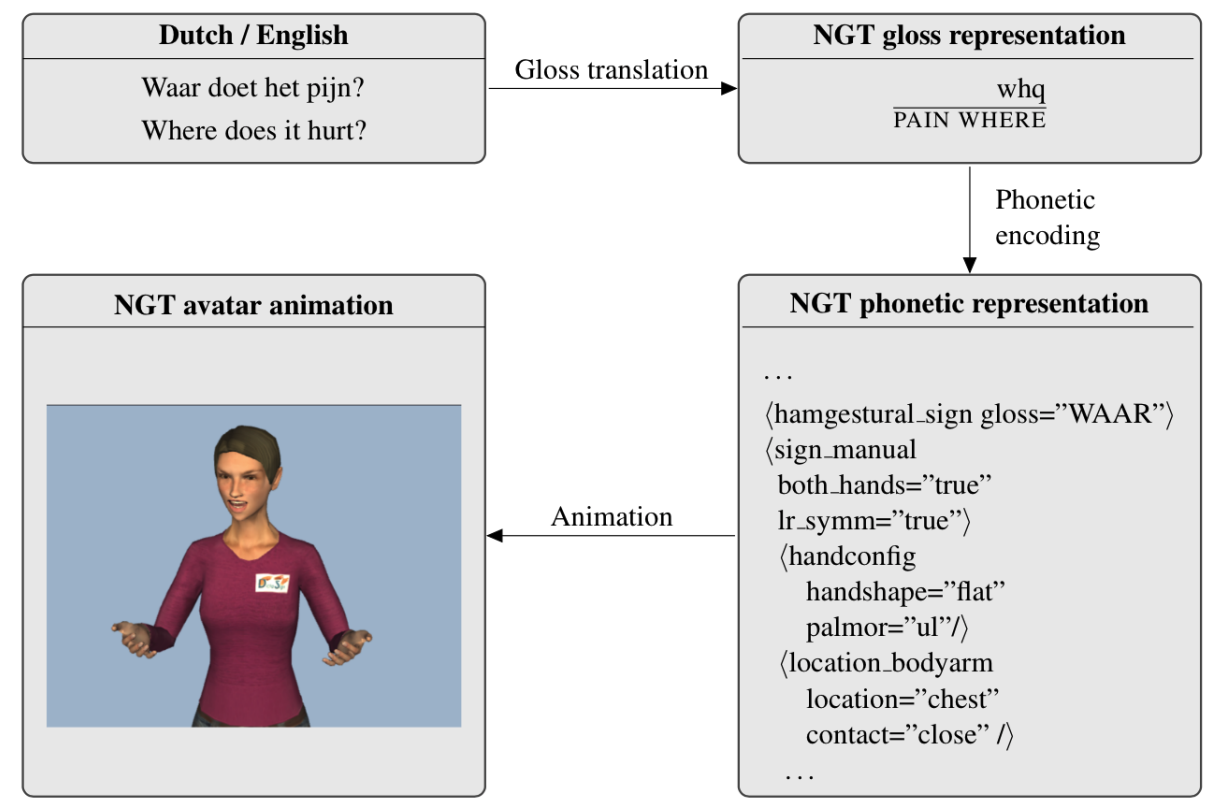

Fig. 2. Overview of the modular translation pipeline.

variation in the actual realisation of 'whq' in NGT [426, furrowed eyebrows are seen as the most canonical realisation [19].

whq

The second step is to map this gloss representation to a phonetic representation in SiGML, a fragment of which is displayed in Figure 2. Finally, this SiGML representation is fed into the JASigning avatar engine, which generates an animation (see Appendix A for a visualisation).

\section{Implementation}

The implementation choices we have made were driven by the specific objective to address the urgent need for a translation tool to aid healthcare professionals in communicating with deaf patients, ensuing from the current pandemic. Two requirements follow from this objective: (i) the system had to be developed within a short time-frame, and (ii) high accuracy of the delivered translations was more important than broad approximate coverage.

Our aim has therefore not been to automate the entire translation process. In particular, automating the process of mapping input sentences to the corresponding NGT glosses using machine learning techniques would not have been feasible within a short time-frame, and would, even in the somewhat longer 
term, most likely result in an unacceptably low accuracy rate for use in a healthcare setting ${ }^{2}$ We therefore mainly focused on automating the phonetic encoding step, something that significantly reduces the manual labor needed in the overall translation pipeline. Automating the mapping from glosses to phonetic representations has not been done in previous work on NGT [23] and, to the best of our knowledge, not in work on other sign languages either.

\subsection{Collecting phrases for translation}

We collected a set of phrases that are commonly used during the diagnosis and treatment of COVID-19, based on consultation with healthcare professionals at the Amsterdam University Medical Centre (AUMC) as well as direct experience (one of the authors is a medical doctor). We also consulted a list of phrases that was used in the SignTranslate system [21] $?^{3}$

The resulting corpus was then divided into three categories: video-only, avataronly, and hybrid. The first category, video-only, consisted mainly of sentences that could be divided into three further categories: emotional, complex, and informed consent. Sentences concerning the patient's emotional well-being require a high level of empathy to be conveyed, which is difficult to achieve in a satisfactory way with an avatar given the current state of the art. We therefore deemed that video translations were necessary for these sentences. Sentences were classified as complex when they involved a combination of several statements and/or questions, or required a demonstration of pictures or diagrams along with an explanation (see Appendix B for an example). Finally, in the case of questions and statements concerning informed consent it is especially important to leave no room for potential misunderstandings. To ensure this, we chose to always offer video translations of these sentences.

The second category, avatar-only, consisted of sentences with many variations differing by only one word or phrase, indicating for instance the time of day or a number of weeks. It would not have been feasible to record a video translation for each version of these sentences.

The third category, hybrid, consisted of sentences that do not fall into one of the other two categories. For these, the system offers both a video translation and an avatar translation. In some cases, the avatar translation is slightly simplified compared to the video translation.

After categorising all of the sentences, those from the first and third category were translated into NGT and recorded by a team consisting of a sign language interpreter and a deaf signer. The deaf signer who is visible in the videos was

\footnotetext{
${ }^{2}$ An recent study investigating the feasibility of automated sign language translation for children television programs in the Netherlands [23] drew the same conclusion.

3 The SignTranslate system was developed in the UK around 2010 to translate phrases common in a healthcare setting from English to British Sign Language. Translations were displayed by means of videos, not by avatar animations. Evidently, the system was not specifically targeted at COVID-19 healthcare. However, many generalpurpose phrases are also relevant in the diagnosis and treatment of COVID-19.
} 
chosen for her clear signing style without a specific dialect, and her neutral reputation within the deaf community. Translations were checked by one of the authors (Smeijers), who is a sign linguist and a medical doctor. This resulted in a collection of 139 video translations. The sentences from the second and third category (including all variations) together comprised 7720 sentences for avatar translation.

\subsection{Constructing SiGML representations}

In order for the system to operate fast at run-time, we pre-processed all sentences and stored SiGML representations of their translations in a database. At run-time, the system only queries this database and does not compute any translations on the fly.

To construct the SiGML representations of full sentences, we implemented a program that, when given the gloss representation of a sentence in NGT, creates the SiGML code for that sentence. It first retrieves the SiGML code for all lexical signs in the given gloss from a lexical database, and then adapts this code to add non-manual grammatical elements. For instance, in the case of yes/no questions, the program makes sure that the sentence ends with the general interrogative sign in NGT (palms up, raised eyebrows) and changes the non-manual component of the last sign before this general interrogative sign to include raised eyebrows, in line with what we observed in our collection of video translations. In the case of wh-questions, the general interrogative sign was also always appended at the end of the sentence. Although the use of this sign in questions is in fact optional in NGT [4, we expect that it increases comprehension in the case of avatar translations.

\subsection{User interface}

We developed an online user interface. The user chooses a translation format (video or avatar) and enters a sequence of search terms. Based on their input they are presented with a list of available sentences from the database. These sentences may differ depending on the translation format chosen (video/avatar). After selecting a sentence the translation is offered in the chosen format.

As mentioned earlier, some of the possible input sentences differ only in one word or phrase. These sentences can be thought of as involving a general template with a variable that can take several values, such as a day of the week, a time of day, or a number of times / minutes / hours / days / weeks / months. When a user wants to translate such a sentence, they first select the template and then provide the intended value for the variable. For example, they may select the template "I am going to explain more at *time*", and then select a particular time (as illustrated in Appendix C).

While JASigning in principle offers a number of different avatars for sign language animation, there are differences in execution between these avatars. Our user interface therefore only makes use of one of the avatars, Francoise, and 
does not allow the user to choose between different options. We intend to further optimise the visualisation of the avatar in future work.

\section{Discussion}

As a first step in evaluating the system we have consulted extensively with a prominent member of the deaf community in the Netherlands who has years of experience in advising organisations (especially museums and hospitals) on how to make their services more accessible to deaf people. Based on these consultations and our own experiences in developing the system, we believe that the following considerations will be helpful in guiding further work in this direction.

The main advantage of avatar technology over video translation is that it provides flexibility and scales up more easily. Once a library of animated signs has been created, and a procedure to integrate non-manual grammatical markers has been implemented, translations for many sentences can be generated. This makes it particularly straightforward to provide translations for sentences that differ only slightly from each other (e.g., in a phrase indicating the time of day).

A disadvantage, however, of avatar translations is that they can be less natural and more difficult to comprehend. While several empirical studies have reported promising comprehension rates for JASigning avatars (see, e.g., [1825]), our consultant indicates that certain avatar translations offered by our system may be difficult to understand for some users. Certain signs differ from each other only in rather subtle ways, and may be indistinguishable when produced by the JASigning avatar. Certain facial expressions and body movements of the avatar are quite unnatural, which can add to the difficulty of understanding translations. Certainly, the avatar's ability to display emotional empathy is very limited. This makes it undesirable to use avatar translations in situations where such empathy is required, as is often the case in medical settings.

Video translations, on the other hand, have their own benefits and drawbacks. They are better than avatar translations in terms of naturalness and comprehensibility, especially in the case of complex sentences. Moreover, our consultant indicates that patients are likely to feel more comfortable watching a video of a human signer rather than an animated avatar in a situation in which their physical well-being is at stake.

The main disadvantage of a video translation system is its inability to scale up efficiently. All translations have to be recorded separately, even ones that are almost identical. Cutting and pasting video fragments of individual signs to create new sentences does not yield satisfactory results.

A general advantage that a machine translation system (using either prerecorded videos, or an avatar, or both) may sometimes have over a human interpreter, especially in the healthcare domain, concerns privacy (see also [3]). A patient may receive sensitive information, and may not want this information to be known to anyone else (the deaf community in the Netherlands is relatively small, which makes it relatively likely that a patient and an interpreter are personally acquainted). In this case, employing a human interpreter has a 
disadvantage (though this may of course be outweighed by the higher level of translation accuracy and empathy that can be provided by a human interpreter).

It is important to emphasise that constructing sign language translations in either format is a time-consuming affair, though for different reasons. Building a corpus of video translations is time intensive because every translation has to be recorded separately. For avatar translations, it takes time to encode individual signs. The latter are reusable, however, which becomes especially attractive as the number of required translations grows. However, the overall preference for one method over another is context-dependent: pros and cons should be carefully weighed in each specific context.

Finally, we note that one clear limitation of the current system is that it only translates text into sign language, not the other way around. This means, for instance, that if a doctor uses the system to ask a deaf patient an open-ended question such as How do you feel?, and if the patient gives an elaborate answer in NGT, the doctor will most likely not be able to understand the answer and our system will not be of help in this case. Overcoming this limitation would require incorporating sign recognition technology (see, e.g., [29]), which has been beyond the scope of our project so far. Note, however, that if a doctor uses our system to ask a more specific yes/no question such as Do you feel dizzy?, then the answer in NGT - involving a head nod in the case of yes and a head shake in the case of no-will most likely be perfectly clear for the doctor even without a general understanding of NGT. Thus, the current system is able to support relatively simple dialogues, but it is limited in scope and certainly does not (yet) offer a full-fledged dialogue system. We view it as a first, but critical step toward a more comprehensive solution.

\section{Conclusion and future work}

We have investigated the potential of automated text-to-sign translation to address the challenges that the current pandemic imposes on the communication between healthcare professionals and deaf patients. We have motivated a modular approach to automated text-to-sign translation, and have built a first prototype system following this approach. We have discussed various prospects and limitations of the system.

In future work, we intend to evaluate the system more thoroughly and to employ the modular approach motivated here to develop text-to-sign translation systems for different domains, e.g., for announcements at airports or railway stations, a use case which has already been explored to some extent for other sign languages [28]. In addition, we also intend to improve the avatar visualisation. 


\section{References}

1. Baker, A., van den Bogaerde, B., Pfau, R., Schermer, T.: The linguistics of sign languages: An introduction. John Benjamins Publishing Company (2016). https://doi.org/10.1075/z.199

2. Battaglino, C., Geraci, C., Lombardo, V., Mazzei, A.: Prototyping and preliminary evaluation of a sign language translation system in the railway domain. In: Antona, M., Stephanidis, C. (eds.) Universal Access in Human-Computer Interaction. pp. 339-350 (2015). https://doi.org/10.1007/978-3-319-20681-3_32

3. Bouillon, P., David, B., Strasly, I., Spechbach, H.: A speech translation system for medical dialogue in sign language - Questionnaire on user perspective of videos and the use of Avatar Technology. In: Proceedings of the 3rd Swiss Conference on Barrier-free Communication (BfC 2020). pp. 46-54 (2021)

4. Coerts, J.: Nonmanual grammatical markers: an analysis of interrogatives, negations and topicalisations in Sign Language of the Netherlands. Ph.D. thesis, University of Amsterdam (1992)

5. Courty, N., Gibet, S.: Why is the creation of a virtual signer challenging computer animation? In: International Conference on Motion in Games. pp. 290-300. Springer (2010). https://doi.org/10.1007/978-3-642-16958-8_27

6. David, B.V.C., Bouillon, P.: Prototype of Automatic Translation to the Sign Language of French-speaking Belgium. Evaluation by the Deaf Community. Modelling, Measurement and Control C 79(4), 162-167 (2018). https://doi.org/10.18280/mmc_c.790402

7. Delorme, M., Filhol, M., Braffort, A.: Animation Generation Process for Sign Language Synthesis. Advances in Computer-Human Interactions, ACHI 9, 386-390 (2009). https://doi.org/10.1109/ACHI.2009.29

8. Ebling, S., Glauert, J.: Building a Swiss German Sign Language avatar with JASigning and evaluating it among the Deaf community. Universal Access in the Information Society 15(4), 577-587 (2016). https://doi.org/10.1007/s10209-0150408-1

9. Elliott, R., Glauert, J., Jennings, V., Kennaway, R.: An overview of the SiGML notation and SiGML signing software system. In: Workshop on the Representation and Processing of Sign Languages at the Fourth International Conference on Language Resources and Evaluation (LREC). pp. 98-104 (2004)

10. Esselink, L.: Lexical resources for sign language synthesis: The translation of Dutch to Sign Language of the Netherlands (2020), bachelor's thesis. University of Amsterdam, https://scripties.uba.uva.nl/search?id=715792

11. Fellinger, J., Holzinger, D., Pollard, R.: Mental health of deaf people. The Lancet 379(9820), 1037-1044 (2012). https://doi.org/10.1016/S0140-6736(11)61143-4

12. Gibet, S., Courty, N., Duarte, K., Naour, T.L.: The SignCom system for datadriven animation of interactive virtual signers: Methodology and evaluation. ACM Transactions on Interactive Intelligent Systems (TiiS) 1(1), 1-23 (2011). https://doi.org/10.1145/2030365.2030371

13. Glauert, J., Elliott, R.: Extending the SiGML notation-a progress report. In: Second International Workshop on Sign Language Translation and Avatar Technology (SLTAT) (2011)

14. Grote, H., Izagaren, F.: COVID-19: The communication needs of D/deaf healthcare workers and patients are being forgotten. British Medical Journal 369 (2020). https://doi.org/10.1136/bmj.m2372 
15. Hanke, T.: Hamnosys-representing sign language data in language resources and language processing contexts. In: LREC. vol. 4, pp. 1-6 (2004)

16. Jennings, V., Elliott, R., Kennaway, R., Glauert, J.: Requirements for a signing avatar. In: Workshop on Corpora and Sign Language Technologies at the 7 th International Conference on Language Resources and Evaluation (LREC 2010). pp. 33-136 (2010)

17. Kelly, L.P., Barac-Cikoja, D.: The comprehension of skilled deaf readers. In: Cain, K., Oakhill, J. (eds.) Children's comprehension problems in oral and written language: A cognitive perspective, pp. 244-280. The Guilford Press (2007)

18. Kennaway, R., Glauert, J., Zwitserlood, I.: Providing signed content on the internet by synthesized animation. ACM Transactions on Computer-Human Interaction 14(3), 1-29 (2007). https://doi.org/10.1145/1279700.1279705

19. Klomp, U.: A descriptive grammar of Sign Language of the Netherlands. Ph.D. thesis, University of Amsterdam (2021)

20. McKee, M., Moran, C., Zazove, P.: Overcoming additional barriers to care for deaf and hard of hearing patients during covid-19. JAMA Otolaryngology-Head \& Neck Surgery 146(9), 781-782 (2020). https://doi.org/10.1001/jamaoto.2020.1705

21. Middleton, A., Niruban, A., Girling, G., Myint, P.K.: Communicating in a healthcare setting with people who have hearing loss. Bmj 341 (2010). https://doi.org/10.1136/bmj.c4672

22. Prillwitz, S., Leven, R., Zienert, H., Hanke, T., Henning, J.: HamNoSys version 2.0: an introductory guide (1989)

23. Prins, M., Janssen, J.B.: Automated sign language (2014), TNO technical report

24. Rayner, M., Bouillon, P., Ebling, S., Gerlach, J., Strasly, I., Tsourakis, N.: An open web platform for rule-based speech-to-sign translation. In: Proceedings of the 54th Annual Meeting of the Association for Computational Linguistics. pp. 162-168. Association for Computational Linguistics, Berlin, Germany (2016). https://doi.org/10.18653/v1/P16-2027, https://www.aclweb.org/ anthology/P16-2027

25. Smith, R.G., Nolan, B.: Emotional facial expressions in synthesised sign language avatars: a manual evaluation. Universal Access in the Information Society 15(4), 567-576 (2016). https://doi.org/10.1007/s10209-015-0410-7

26. de Vos, C., van der Kooij, E., Crasborn, O.: Mixed signals: Combining linguistic and affective functions of eyebrows in questions in Sign Language of the Netherlands. Language and Speech 52(2-3), 315-339 (2009). https://doi.org/10.1177/0023830909103177

27. Wauters, L.N., Van Bon, W.H., Tellings, A.E.: Reading comprehension of Dutch deaf children. Reading and writing 19(1), 49-76 (2006). https://doi.org/10.1007/s11145-004-5894-0

28. Wolfe, R., Cook, P., McDonald, J.C., Schnepp, J.: Linguistics as structure in computer animation: Toward a more effective synthesis of brow motion in American Sign Language. Sign Language \& Linguistics 14(1), 179-199 (2011). https://doi.org/10.1075/sll.14.1.09wol

29. Zhou, Z., Chen, K., Li, X., Zhang, S., Wu, Y., Zhou, Y., Meng, K., Sun, C., He, Q., Fan, W.: Sign-to-speech translation using machine-learningassisted stretchable sensor arrays. Nature Electronics 3(9), 571-578 (2020). https://doi.org/10.1038/s41928-020-0428-6

30. Zwitserlood, I.: Synthetic signing. In: The World of Content Creation, Management, and Delivery (IBC 2005). p. 352-357 (2005) 
A UI example: avatar translation of a simple question

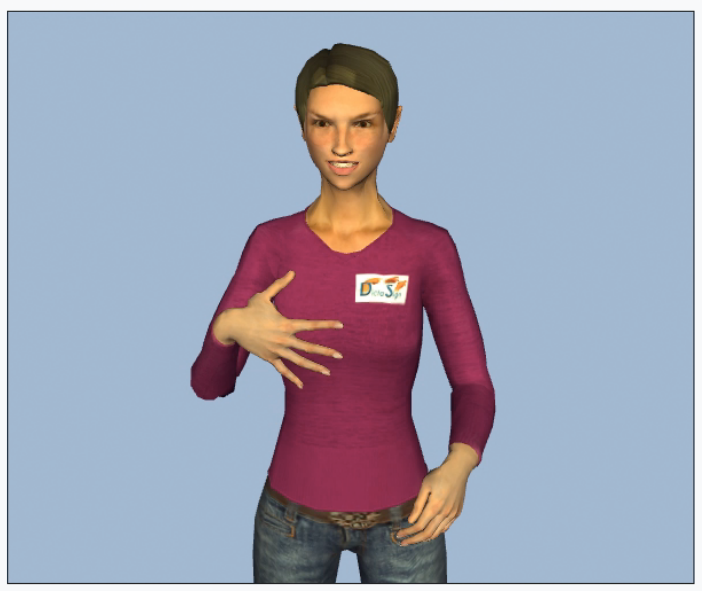

Choose a translation option:

Video translation

- Avatar translation

Where are you feeling pain?

\section{PLAY}

Adjust speed: $+0.0+-+$

Gloss: pijn

B UI example: video translation of a complex question

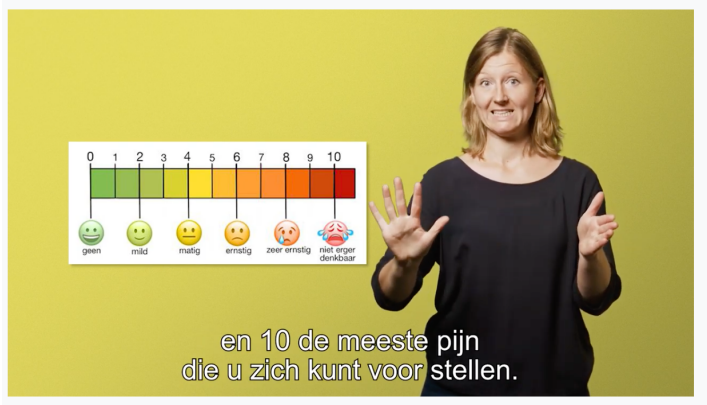

Choose a translation option:

- Video translation

Avatar translation

On a scale of $0-10$, where 0 is 'no pain' and 10 is the

worst pain you can imagine, how much pain are you

feeling? 


\section{UI example: translation of a sentence with a variable}

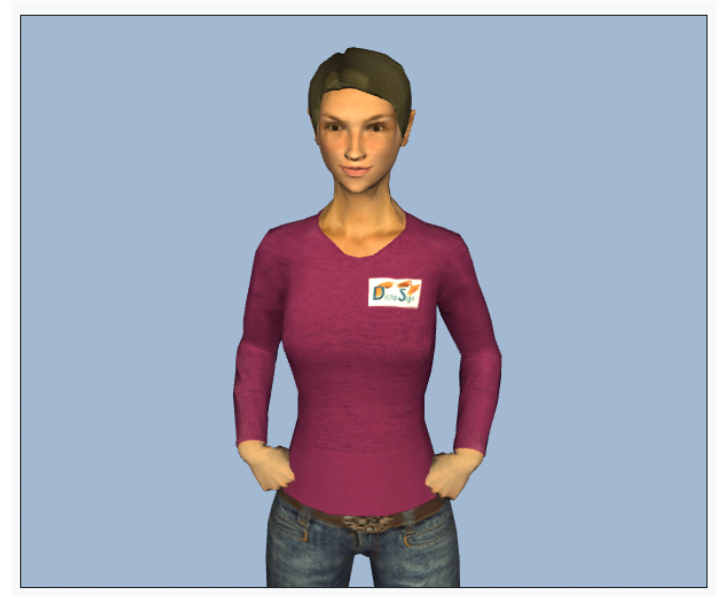

Choose a translation option:

Video translation

- Avatar translation

I am going to explain more at *time*.

15:10| Submit

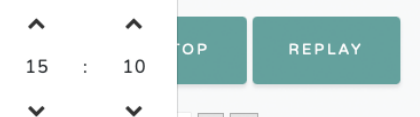

Gloss: standaard_pose

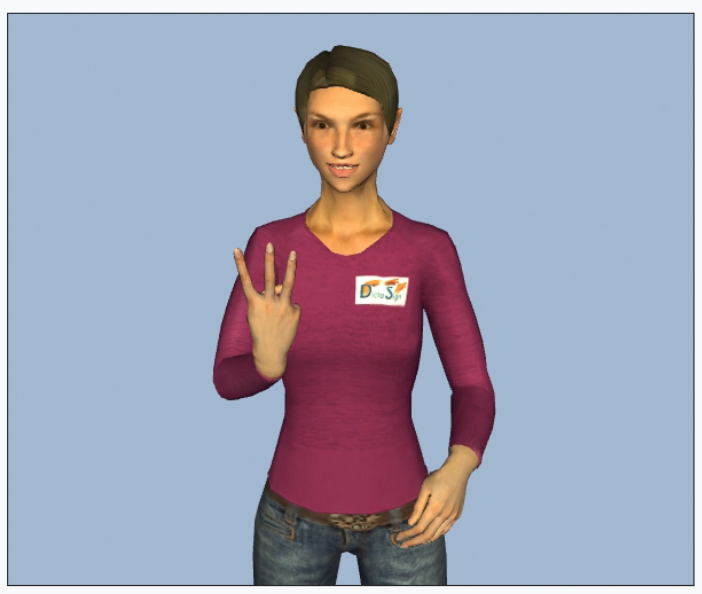

Choose a translation option:

Video translation

- Avatar translation

I am going to explain more at 10 past 3 in the

afternoon.

STOP

REPLAY

Adjust speed: $+0.0 \quad-+$

Gloss:

drie_3 